\title{
La evaluación del docente de matemática y su incidencia en los procesos de enseñanza aprendizaje. @(1)(
}

\section{The evaluation of the teacher of mathematics and its incidence in the processes of teaching and learning.}

Juan Carlos Sarmiento Saavedra, MSc. ${ }^{1}$, Troya Loor María Angélica. ${ }^{2}$, Núñez Freire Luis Alfonso MAE. ${ }^{3}$ \& Eugenio Rafael Mora Zambrano. ${ }^{4}$

Recibido: 02-09-2017 / Revisado:03-11-2017 Aceptado: 10-12-2017/ Publicado: 01-01-2018

\begin{abstract}
.
DOI: $\underline{\text { https://doi.org/10.33262/cienciadigital.v2i1.16 }}$
\end{abstract}

Education is a right that all people have at the level of all the nations of the world and therefore at a Latin American level, which seeks the development and improvement of the standard of living, in this way to stop being underdeveloped and dependent countries. For this reason the education given by teachers in conjunction with authorities and administrative staff of schools should be of quality and should lead us to a constant evaluation of teaching-learning processes, emphasizing in the teaching and administrative process, the Colegio Nacional Mixto Santo Domingo de los Colorados, is a middle school establishment that operates in the city of the same name. the academic formation is constantly innovating, the applied research is Field, with a descriptive method, taking into account the direct contact with the object, a sample and interview was applied to 22 teachers, 22 students and 74 parents, with this evaluation that in its essence it seeks the permanent and integral analysis of the student not so that he consciously assumes his successes and errors; it has become the measurement of the repetition of what has been taught by the teacher, transforming itself into a purely

\footnotetext{
${ }^{1}$ Universidad Técnica Luis Vargas Torres de Esmeraldas Ext. la Concordia,jan_carlos_ss@hotmail.com

${ }^{2}$ Unidad Educativa Dr. Andrés F. Córdova, Esmeraldas, Ecuador, troyangelica@ hotmail.com

${ }^{3}$ Universidad Técnica Luis Vargas Torres de Esmeraldas Ext. la Concordia, luis.nunez@utelvt.edu.ec

${ }^{4}$ Instituto Tecnológico superior Japón, Esmeraldas, Ecuador, geniomora@hotmail.com
} 
quantitative evaluation that seeks the promotion of the student at the scientific level, product of the vision that teachers have in this regard

Keywords: Mathematics, Learning Process, Evaluation Of Education, Secondary School Teacher.

\section{Resumen.}

La educación es un derecho que tienen todas personas a nivel de todas las naciones del mundo y por ende a nivel latinoamericano, la cual busca el desarrollo y mejoramiento del nivel de vida, de esta manera dejar de ser países subdesarrollados y dependientes. Por tal motivo la educación impartida por docentes en conjunto con autoridades y administrativos de los planteles educativos debe ser de calidad y nos debería conducir a una constante evaluación de los procesos de enseñanza aprendizaje, enfatizando en el proceso docente y administrativo, el Colegio Nacional Mixto Santo Domingo de los Colorados, es un establecimiento de educación media que funciona en la ciudad del mismo nombre. la formación académica se está innovando constantemente, la investigación aplicada es de Campo, con un método descriptivo, tomando en cuenta el contacto directo con el objeto, se aplicó un muestra y entrevista a 22 docentes, 22 estudiantes y74 padres de familia, con esta evaluación que en su esencia busca el análisis permanente e integral del estudiante no para que de manera consciente asuma sus aciertos y errores; se ha convertido en la medición de la repetición de lo enseñado por el profesor transformándose en una evaluación netamente cuantitativa que busca la promoción del estudiante a nivel científico, producto de la visión que los maestros tienen al respecto.

Palabras Claves: Matemáticas, Proceso de aprendizaje, Evaluación de la educación, Docente de secundaria 


\section{Introducción.}

La educación es un derecho que tienen todas personas a nivel de todas las naciones del mundo y por ende a nivel latinoamericano, la cual busca el desarrollo y mejoramiento del nivel de vida, de esta manera dejar de ser países subdesarrollados y dependientes. Por tal motivo la educación impartida por docentes en conjunto con autoridades y administrativos de los planteles educativos debe ser de calidad y nos debería conducir a una constante evaluación de los procesos de enseñanza aprendizaje, enfatizando en el proceso docente y administrativo (Becerra \& Moya, 2008).

La deficiencia en la evaluación para los establecimientos educativos de la provincia sea por la resistencia a ser evaluados los docentes o actualización de los mismo, no contribuyen a obtener una educación de calidad en los estudiantes de manera general. El Colegio Nacional Mixto Santo Domingo de los Colorados, es un establecimiento de educación media que funciona en la ciudad del mismo nombre con sujeción a la Ley y Reglamento de Carrera Docente y escalafón del Ministerio Nacional; a la Ley y reglamento General de la Ley de Educación y Cultura, al Vademécum Legal de la Contraloría General del Estado, Ley y Presupuesto y su Reglamento; manual Administrativo y Financiero para Colegios e Instituciones para Colegios e Institutos Superiores y Fiscales; Reglamentos de Orientación Educativa y Bienestar y al Reglamento Interno del Colegio (MINEDUC, 2015).

Además, el Colegio está motivando la formación académica e innovando constantemente, sin embargo, los fundamentos psicopedagógicos, sociológicos y filosóficos que permiten optimizar el proceso de aprendizaje, no han tenido en algunos de nuestros profesores una correcta aplicación, lo cual ha impedido un mejor desenvolvimiento de la labor educativa (SEMPLADES, 2017). La evaluación que en su esencia busca el análisis permanente e integral del estudiante no para que de manera consciente asuma sus aciertos y errores; se ha convertido en la medición de la repetición de lo enseñado por el profesor transformándose en una evaluación netamente cuantitativa que busca la promoción del estudiante a nivel científico, producto de la visión que los maestros tienen al respecto (Devia \& Pinilla, 2012) 
La evaluación juzga tanto el proceso de aprendizaje y desarrollo del currículo como los logros de los estudiantes. En este sentido, una diferencia fundamental con respecto al término tradicional de los exámenes (prueba oral o escrita fijada en un tiempo y muy controlada), la evaluación continua, que se realiza con otro tipo de medios, entre los que se incluye el conjunto de tareas realizadas por el estudiante durante el curso. Así, la evaluación se realiza generalmente para obtener una información global y envolvente de las actividades y no la simple y puntual referencia de los papeles escritos en el momento del examen (Alpizar, 2014)

La evaluación institucional cumple una función legitimadora de la posición de las instituciones educativa en lo que respecta al desarrollo del currículo institucional que permita mejorar el proceso de enseñanza aprendizaje y elevar la calidad del docente y administrativos de una institución, en base a lo que necesitan las sociedades modernas, al proporcionar un mecanismo por el cual se hacen juicios sobre el mérito, al mismo tiempo que ayuda a definir el mismo concepto de mérito en las sociedades modernas. Los buenos resultados académicos se aceptan como un indicador de las habilidades que permitirán a un individuo progresar y tener éxito en una sociedad que a su vez seleccionará a aquellos que contribuirán más en ella, en términos de liderazgo social y económico (Matas \& Quispe, 2014).

La educación considerada como instrumento principal de progreso y desarrollo de los pueblos, se ha visto limitada por la desatención que le prestan las autoridades de turno, además algunos educadores tienen una actitud conformista no tratan de superar las dificultades para lograr una educación de calidad, basada en una coordinación académica curricular de parte de los docentes y administrativos de una institución. La cultura de la evaluación institucional no ha logrado un impacto adecuado en la educación, en particular, en el Colegio de Bachillerato. Se ha centrado fundamentalmente en reportar indicadores tradicionales de: cobertura, índices de aprobación, reprobación o deserción. La evaluación al Docente del Área de Matemática no se realiza de acuerdo con los aspectos académicos que exigen la asimilación y aprendizaje del idioma extranjero en los procesos de enseñanza aprendizaje (Rodriguez, 2010). 
No se valora adecuadamente elementos de la gestión como son la actuación y el liderazgo de sus directivos, el logro de objetivos, los apoyos académicos y administrativos, el logro de los programas propuestos, así como de la misión institucional (Alpizar, 2014). La evaluación de la educación se ha venido desarrollando de una manera desvinculada, ajena a las decisiones políticas que se dan respecto a la educación; y en el mejor de los casos como un procedimiento utilizado para confirmar la certeza de la decisión tomada por las autoridades. Determinar de qué manera incide la falta de evaluación al Docente del Área de Matemática en el mejoramiento del proceso enseñanza aprendizaje, con el fin de concienciar a los docentes del área de matemática de la importancia que tiene la evaluación en el proceso de enseñanza aprendizaje del Colegio Nacional Mixto "Santo Domingo de los Colorados” de la ciudad de Santo Domingo, provincia de los Tsáchilas (Pérez, 2011).

La evaluación educacional se ha desarrollado, más por razones sociales que educacionales, para facilitar la selección social y económica y no tanto por motivos educacionales propiamente dichos (Asamblea Nacional, 2008). Sin embargo, recientemente el interés se ha centrado en paliar los efectos negativos en el sistema educativo y su repercusión en los estudiantes, basados en una evaluación de tipo institucional y curricular en aras de hacerla motivadora controlada en sus procesos de aprendizaje. La política y la práctica de la evaluación siempre incluirán transacciones y compromisos.

Es importante que los docentes comprendan que la evaluación debe considerarse como un elemento importante en el proceso educativo. Por ello se debe sistematizar al proceso evaluativo y hacer de un instrumento imprescindible en la práctica pedagógica, por tal motivo esta investigación beneficiará a la institución educativa en todas sus áreas y en especial a los estudiantes, ayudando a mejorar la calidad de la educación en el plantel y de esta manera tener alternativas de solución a un problema tan complejo y delicado como es la evaluación al docente (Guaman \& Cevallos , 2016).

\section{Métodos y materiales.}

Se basa en una Investigación de Campo, con un método descriptivo, tomando en cuenta el contacto directo con el objeto, se aplicó un muestra y entrevista a 22 docentes, 22 estudiantes 
y74 padres de familia, La recolección de la información se basa en la correcta selección del instrumento, la aplicación de la misma se hará de manera distinta tanto para administrativos, docentes y estudiantes, para proceder con la medición y obtener resultados para luego ser analizados con las herramientas seleccionada para el procesamiento de la información como fueron la hoja de cálculo de Microsoft Excel, formulas, tablas, para la tabulación y análisis de datos.

\section{Análisis de resultados.}

Encuesta a los estudiantes:

TABLA 1. ¿CREE USTED QUE SE DESARROLLAN LAS UNIDADES PROGRAMADAS EN MATEMÁTICA EN SU TOTALIDAD?

\begin{tabular}{ccc}
\hline ALTERNATIVA & FRECUENCIA & PORCENTAJE \% \\
\hline SI & 38 & 51 \\
NO & 36 & 49 \\
TOTAL & 74 & 100 \\
\hline
\end{tabular}

Elaborado por: Grupo de Investigación

Los estudiantes piensan que no se desarrollan a cabalidad las unidades programadas (36) que equivale al $49 \%$ y que si 38 estudiantes un $51 \%$.

TABLA 2. ¿LOS DOCENTES DE MATEMÁTICA NECESITAN CAPACITACIÓN PARA DESARROLLAR MEJOR SU FUNCIÓN DENTRO DE LA INSTITUCIÓN?

\begin{tabular}{ccc}
\hline ALTERNATIVA & FRECUENCIA & PORCENTAJE \% \\
\hline SI & 35 & 47 \\
NO & 39 & 53 \\
TOTAL & 74 & 100 \\
\hline
\end{tabular}

Elaborado por: Grupo de Investigación

Los docentes necesitan capacitación un $47 \%$ equivalente a 35 y 39 estudiantes manifiestan que no necesitan equivalente al 53\%. 
TABLA 3. ¿CON QUÉ FRECUENCIA LOS DOCENTES DE MATEMÁTICA LE REALIZAN A USTED EVALUACIÓN?

\begin{tabular}{ccc}
\hline CATEGORÍA & FRECUENCIA & PORCENTAJE \% \\
\hline DIARIO & 8 & 11 \\
SEMANAL & 24 & 32 \\
MENSUAL & 42 & 57 \\
TOTAL & 74 & 100 \\
\hline
\end{tabular}

Elaborado por: Grupo de Investigación.

La evaluación que se le realiza a los estudiantes es mensual lo dicen (42) el 57\% y semanal (24) el 32\%, sólo (8) el 11\% dicen que lo hacen periódicamente como debería ser.

TABLA 4. ¿CON QUE FRECUENCIA CREE USTED QUE LES HAN REALIZADO UNA EVALUACIÓN A LOS DOCENTES DE MATEMÁTICA CON RESPECTO A SUS ACTIVIDADES?

\begin{tabular}{ccc}
\hline CATEGORIA & FRECUENCIA & PORCENTAJE \% \\
\hline MENSUAL & 9 & 12 \\
SEMESTRAL & 14 & 19 \\
ANUAL & 17 & 23 \\
NUNCA & 34 & 46 \\
TOTAL & 74 & 100 \\
\hline
\end{tabular}

Elaborado por: Grupo de Investigación.

Los estudiantes creen que nunca se ha realizado una evaluación a los docentes (34) el 50\%, $17(23 \%)$ dicen que se los evalúa anualmente y 14 que se los ha evaluado semestralmente.

Encuesta a los padres de familia:

TABla 5. LAS ASIGNATURAS QUE LE DICTAN A SU HIJO ESTÁN DE ACUERDO CON SU ESPECIALIDAD.

\begin{tabular}{ccc}
\hline CATEGORÍA & FRECUENCIA & PORCENTAJE \% \\
\hline SI & 56 & 76 \\
NO & 18 & 24 \\
TOTAL & 74 & 100 \\
\hline
\end{tabular}

Elaborado por: Grupo de Investigación. 
El 76\% (56) de los padres de familia están de acuerdo con las asignaturas que les dictan a sus hijos y el 24\% (18) no están de acuerdo.

TABLA 6. ¿CREE UD. QUE LOS DOCENTES DE MATEMÁTICA NECESITAN CAPACITACIÓN PARA DESARROLLAR MEJOR SU FUNCIÓN DENTRO DE LA INSTITUCIÓN?

\begin{tabular}{ccc}
\hline CATEGORÍA & FRECUENCIA & PORCENTAJE \% \\
\hline SI & 67 & 90 \\
NO & 7 & 10 \\
TOTAL & 74 & 100 \\
\hline
\end{tabular}

Elaborado por: Grupo de Investigación.

Es así que el 90\% (67) de los padres de familia manifiestan que el personal en general necesita capacitación y apenas el 10\% (7) manifiestan que no necesitan capacitación.

TABLA 7. ¿CREE USTED QUE LES HAN REALIZADO UNA EVALUACIÓN A LOS DOCENTES DE MATEMÁTICA DE SUS ACTIVIDADES?

\begin{tabular}{ccc}
\hline CATEGORÍA & FRECUENCIA & PORCENTAJE \% \\
\hline SI & 47 & 64 \\
NO & 27 & 36 \\
TOTAL & 74 & 100 \\
\hline
\end{tabular}

Elaborado por: Grupo de Investigación.

El 64\% (47) de los padres de familia creen que han realizado evaluación a los docentes y administrativos y el 36\% (27) dicen que no han realizado evaluación al personal.

Encuesta a los docentes.

TABLA 8. ¿CON QUE FRECUENCIA RECIBE USTED CAPACITACIÓN ACORDE CON SU FUNCIÓN DENTRO DE LA INSTITUCIÓN QUE LABORA?

\begin{tabular}{ccc}
\hline CATEGORÍA & FRECUENCIA & PORCENTAJE \% \\
\hline SEMESTRAL & 0 & 0 \\
ANUAL & 14 & 64 \\
C/ DOS AÑOS & 2 & 9 \\
NUNCA & 6 & 27 \\
TOTAL & $\mathbf{2 2}$ & $\mathbf{1 0 0}$ \\
\hline
\end{tabular}

Elaborado por: Grupo de Investigación. 
El 64\% (14) manifiesta que reciben capacitación acorde con su función anualmente, el 9\%

(2) indica que recibe cada dos años y el $27 \%$ (6) dicen que nunca han recibido capacitación acorde con su función.

TABLA 9. ¿DESARROLLÓ USTED LAS UNIDADES PROGRAMADAS EN SU TOTALIDAD?

\begin{tabular}{ccc}
\hline CATEGORÍA & FRECUENCIA & PORCENTAJE \% \\
\hline SI & 14 & 64 \\
NO & 8 & 36 \\
TOTAL & $\mathbf{2 2}$ & $\mathbf{1 0 0}$ \\
\hline
\end{tabular}

Elaborado por: Grupo de Investigación.

El 64\% (14) desarrolla las actividades programadas en su totalidad y el 36\% (8) no las desarrolla en su totalidad.

TABLA 10. ¿NECESITA USTED CAPACITACIÓN PARA DESARROLlaR ALGÚN TEMA EN PARTICULAR QUE ESTÉ EN SU PLANIFICACIÓN?

\begin{tabular}{ccc}
\hline CATEGORÍA & FRECUENCIA & PORCENTAJE \% \\
\hline SI & 14 & 64 \\
NO & 8 & 36 \\
TOTAL & $\mathbf{2 2}$ & $\mathbf{1 0 0}$ \\
\hline
\end{tabular}

Elaborado por: Grupo de Investigación.

Se evidencia que al docente y administrativo le falta capacitación debido a que no actualiza sus conocimientos para realizar mejor su trabajo y lograr una educación de calidad.

TABLA 11. ¿CON QUE FRECUENCIA REALIZA USTED EVALUACIÓN A SUS ESTUDIANTES?

\begin{tabular}{ccc}
\hline CATEGORÍA & FRECUENCIA & PORCENTAJE \% \\
\hline DIARIO & 5 & 23 \\
SEMANAL & 7 & 32 \\
MENSUAL & 10 & 45 \\
TOTAL & $\mathbf{2 2}$ & $\mathbf{1 0 0}$ \\
\hline
\end{tabular}

Elaborado por: Grupo de Investigación

Las evaluaciones a los estudiantes según los docentes encuestados las hacen mensualmente un $45 \%$ (10), semanalmente el $32 \%$ (7) y a diario el $23 \%$ (5). 
TABLA 12. ¿CON QUE FRECUENCIA LE HAN REALIZADO EVALUACIÓN DE SUS ACTIVIDADES EDUCATIVAS?

\begin{tabular}{ccc}
\hline CATEGORÍA & FRECUENCIA & PORCENTAJE \% \\
\hline MENSUAL & 0 & 0 \\
SEMESTRAL & 2 & 9 \\
ANUAL & 8 & 36 \\
NUNCA & 12 & 55 \\
TOTAL & $\mathbf{2 2}$ & $\mathbf{1 0 0}$ \\
\hline
\end{tabular}

Elaborado por: Grupo de Investigación.

Los docentes y administrativos indican que nunca le han realizado una evaluación en un 55\% (12), que los han evaluado anualmente el 36\% (8) y semestralmente el 9\% (2).

TABLA 13. ¿LE GUSTARÍA QUE LO EVALÚEN?

\begin{tabular}{ccc}
\hline CATEGORIA & FRECUENCIA & PORCENTAJE \% \\
\hline AUTORIDADES & 5 & 23 \\
ESTUDIANTES & 12 & 54 \\
NINGUNO & 5 & 23 \\
TOTAL & $\mathbf{2 2}$ & $\mathbf{1 0 0}$ \\
\hline
\end{tabular}

Elaborado por: Grupo de Investigación

Los docentes y administrativos quisieran que los evalúen las autoridades el $23 \%$ (5), los estudiantes el $54 \%$ (12) y ninguno de los dos el $23 \%$ (5).

\section{Discusión.}

Se evidencia de esta manera un descontento por parte de los estudiantes ya que no pueden nutrirse de los conocimientos necesarios los cuales son básicos para su especialidad. Los mismos manifiestan que el personal que labora en la institución necesita de una capacitación para desarrollar de mejor manera sus actividades y poder satisfacer todas las inquietudes que se presentan dentro del plantel en el desarrollo de la actividad docente. Con esto se entiende que necesariamente hay que evaluar al personal de la institución, esto indica que no se verifica si se asimilan o no los conocimientos y por ende no se puede saber qué es lo que hay que reforzar y proceder a una retroalimentación de los temas más complejos. Lo que significa 
que los docentes necesitan ser evaluados para conocer si están actualizados o no, valorar la eficiencia y eficacia que permita desarrollar e impartir una educación de calidad.

Es importante que se aplique permanentemente evaluaciones a los docentes y administrativos, esto permitirá verificar el nivel académico de cada uno de ellos y un mejor control de las planificaciones en clase y desarrollar las unidades programadas en su totalidad, para así elevar el nivel de enseñanza-aprendizaje y motivación en los estudiantes.

Los padres de familia sienten conformidad con las asignaturas que conforman el currículo de la especialidad, no obstante, el personal docente como el administrativo necesita capacitación para desarrollar sus actividades pedagógicas y mejorar el proceso de enseñanza - aprendizaje. Esto se debe a que por diversos motivos no se pueden cumplir (perdidas de clases, actividades extra curriculares, etc.), las actividades de capacitación que se realicen en la institución deben de estar dirigidas de acuerdo con la especialidad y a la función que realiza el personal.

\section{Conclusiones.}

- Una vez realizada la investigación puedo concluir diciendo que la evaluación de la gestión debe formar parte integral de la evaluación institucional. El directivo, dentro de su función de llevar a la institución al logro de sus fines o misión, tiene un deber jurídico y moral de rendir cuentas y a su vez, de ser evaluado. La evaluación debe ser un proceso compartido, en equipo y convertirse en un proceso de aprendizaje permanente. De acuerdo con las encuestas realizadas y analizando el sentir de los estudiantes y por qué no mejora el nivel académico puedo atribuir a las siguientes causas:

- Los estudiantes concuerdan en que hay asignaturas que se deberían dictar como diseño gráfico el $24 \%$ y dibujo técnico el $30 \%$, lo cual ayudaría en el desarrollo de la parte psicomotriz de ellos y se dicta por ejemplo cívica que se debería dar en la educación básica. Los temas que aparecen por necesidad y de interés para los estudiantes no son tratados a fondo y concuerdan el $57 \%$ que los temas son tratados de manera general y el $34 \%$ que los temas de interés son tratados de manera 
superficial. Se deduce la falta de actualización de los docentes de Matemática en un 47\% según dicen los estudiantes. La evaluación de los conocimientos debe ser periódicamente y no mensuales.

- Los padres de familia asumen que no se desarrollan las unidades programadas a cabalidad en un $66 \%$ debido a pérdidas de clases, por falta de capacitación a los docentes en un $90 \%$ y porque no se evalúan los procesos de enseñanza impartidos por los docentes en un 37\%. Los conocimientos desactualizados y el conformismo en el nivel académico del docente el $4.5 \%$ tienen cuarto nivel y el 68\% tienen tercer nivel, la asignación de la cátedra no acorde con su especialidad en un $27 \%$ hace que siga una planificación rígida dada por el área en un $68 \%$ y no puede adaptar temas de trascendencia a la programación curricular.

La falta de evaluación al docente de Matemática en un 55\%, produce descuido en su capacitación y que vaya en beneficio de los estudiantes. El personal administrativo no está siendo capacitado en un $64 \%$ para que realice una mejor labor dentro de la institución y está ubicado en algunos casos en funciones ajenas a su especialidad

\section{Referencias bibliográficas.}

Alpizar, M. (2014). Actitudes del docente de matemáticas de enseñanza secundaria (Eso y bachillerato) en la relación docente-estudiante. Tesis Doctoral Universidat Autónoma de Barcelona, 222. Bellaterra, España. Obtenido de http://www.tdx.cat/bitstream/handle/10803/133226/maar1de1.pdf?sequence=1

Asamblea Nacional. (3 de julio de 2008). Constitucion de la República del Ecuador 2008. Obtenido de oas.org: http://www.oas.org/juridico/pdfs/mesicic4_ecu_const.PDF

Becerra, R., \& Moya, A. (2008). Una perspectiva crítica de la evaluación en matemática en la Educación Superior. Revista Universitaria de Investigación, 9(1), 35. Obtenido de http://www.redalyc.org/articulo.oa?id=41011135002

Devia, R., \& Pinilla, C. (2012). La enseñanza de la matemática: de la formación al trabajo de aula. Educere, 16(55), 13. Obtenido de http://www.redalyc.org/pdf/356/35626140019.pdf 
Guaman , R., \& Cevallos , G. (2016). Análisis comparativo de estilos de aprendizaje y sus niveles de preferencia. Atlante. Cuadernos de Educación y Desarrollo, 14. Obtenido de http://hdl.handle.net/20.500.11763/ATLANTE-estilos

Matas , A., \& Quispe, W. (2014). Evaluación de las “Competencias Docentes" En Maestros de Matemáticas de Puno (Perú). Revista de Currículum y Formación de Profesorado, 18(1), 23. Obtenido de http://www.redalyc.org/articulo.oa?id=56730662015

MINEDUC. (abril de 2015). Ley Orgánica de Educación Intercultural. Obtenido de Ministerio de Educacion del Ecuador : https://educacion.gob.ec/wpcontent/uploads/downloads/2017/02/Ley_Organica_de_Educacion_Intercultural_L OEI_codificado.pdf

Miranda, C., Wilhelm, K., Martin, G., Arancibia, M., \& Osses, S. (2013). Autoestima profesional en docentes beneficiarios del Programa de Postítulo de matemáticas en el contexto de la evaluación docente. Estudios Pedagógicos, 39(1), 15. Obtenido de http://www.redalyc.org/pdf/1735/173528596007.pdf

Monrroy, B. (20 de 12 de 2012). Desempeño Docente y Rendimiento Académico en Matemática de los alumnos de una Institución Educativa de Ventanilla. Tesis e Postgrado de la Universidad de San Ignacio de Loyola. Lima, Perú. Obtenido de http://repositorio.usil.edu.pe/bitstream/123456789/1220/1/2012_Monrroy_Desempe $\% \mathrm{C} 3 \% \mathrm{~B} 1 \mathrm{o} \% 20$ docente $\% 20 \mathrm{y} \% 20$ rendimiento $\% 20 \mathrm{acad} \% \mathrm{C} 3 \%$ A9mico $\% 20 \mathrm{en} \% 20 \mathrm{~m}$ atem $\%$ C3\%A1tica\%20de $\% 2010$ \% $\% 20$ alumnos $\% 20 \mathrm{de} \% 20$ una $\% 20$ instituci\% $\% 3 \%$ B n\%20educativa\%20de\%20Ventanilla-Callao.pdf

Pérez, E. (29 de Agosto de 2011). Sistema de Evaluación Docente para la Carrera de Ingeniería en Sistemas Computacionales. Tesis de PreGrado de la Universidad de Guayaquil. Guayaquil, Guayas, Ecuador. Obtenido de http://repositorio.ug.edu.ec/bitstream/redug/2549/1/Tesis\%20Evaluacion\%20Docen te.pdf

Rodriguez, M. (2010). El perfil del docente de matemática: visión desde la triada matemática cotidianidad y pedagogía integral. "Actualidades Investigativas en Educación", 10(3), 21. Obtenido de http://www.redalyc.org/pdf/447/44717980018.pdf

SEMPLADES. (2017). Secretaria Nacional del Planificacion y desarrollo. Obtenido de Semplades: http://www.planificacion.gob.ec/wpcontent/uploads/downloads/2017/10/PNBV-26-OCT-FINAL_0K.compressed1.pdf 
Para citar el artículo indexado.

Sarmiento J., Troya M., Núñez L. \& Mora E. (2018). La evaluación del docente de matemática y su incidencia en los procesos de enseñanza aprendizaje. Revista electrónica Ciencia Digital 2(1), 223-234. Recuperado desde:

http://www.cienciadigital.org/revistascienciadigital2/index.php/CienciaDigital/article/view $\underline{116 / 16}$

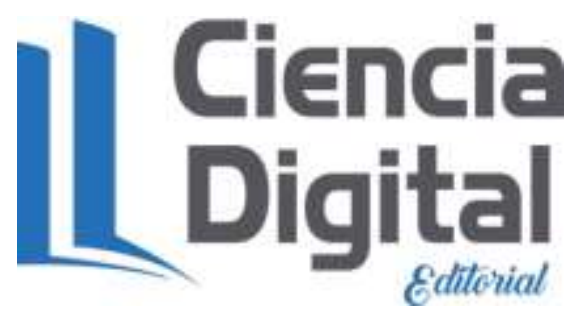

El artículo que se publica es de exclusiva responsabilidad de los autores y no necesariamente reflejan el pensamiento de la Revista Ciencia Digital.

El articulo queda en propiedad de la revista y, por tanto, su publicación parcial y/o total en otro medio tiene que ser autorizado por el director de la Revista Ciencia Digital.
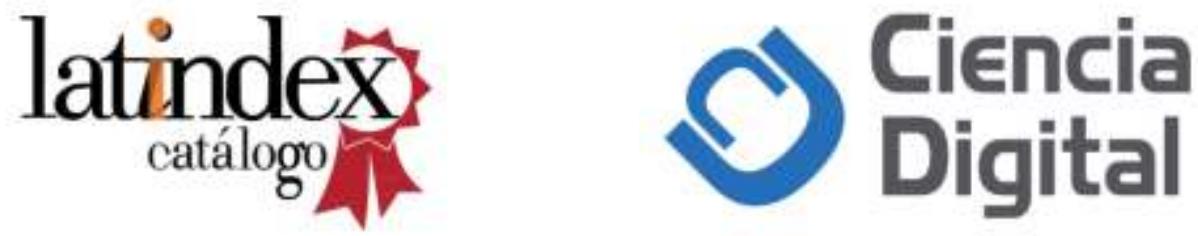